\title{
Predicting the Limits of Atomic Nuclei
}

\author{
First-principles calculations predict the properties of nearly 700 isotopes \\ between helium and iron, showing which nuclides can exist and which \\ cannot.
}

By Marric Stephens

A mong the vast number of possible nuclear isotopes, very few are stable. Stray above a certain mass number-by adding neutrons to an element in the Periodic Table-and eventually the corresponding nucleus can't exist because it leaks nucleons. The neutron "dripline" that defines this limit of existence has been discovered experimentally for elements up to neon (see Viewpoint: Reaching the Limits of Nuclear Existence). Now, using a first-principles theoretical approach, Ragnar Stroberg from the University of Washington, Seattle, and colleagues have predicted the map of nuclear existence as far as iron [1].

This study is not the first attempt to explore the extreme neutron-rich region of the nuclear landscape theoretically: a previous investigation used approximative methods to predict about 7000 bound nuclides between helium and element 120 . Stroberg and colleagues, however, obtained a first map based on $a b$ initio nuclear theory. Starting from two- and

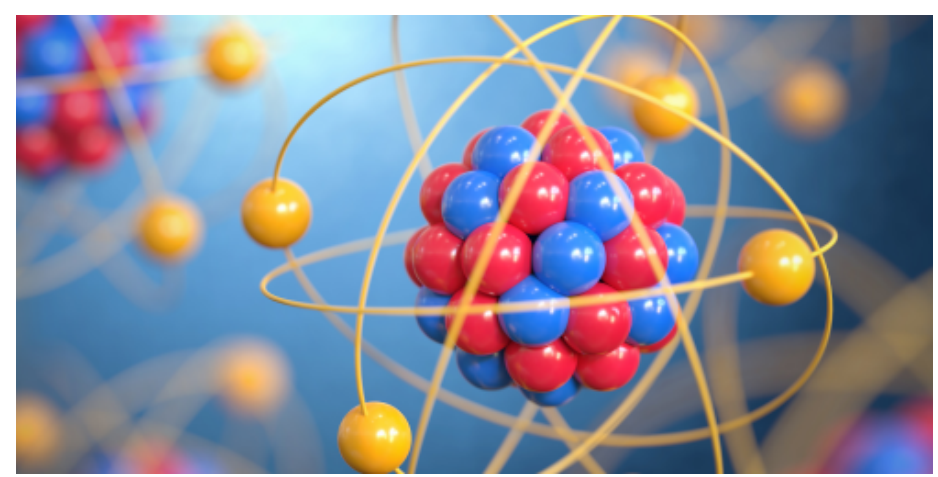

Credit: koya979/adobe.stock.com three-nucleon interactions, they solved the many-body Schrödinger equation to predict properties of nuclei up to iron. To reliably treat light- to medium-mass elements, they combined a novel ab initio many-body method, called the in-medium similarity renormalization group, with an extension that can handle partially filled nuclear orbitals.

The team calculated ground-state energies for nearly 700 isotopes. The resulting energies, which agree remarkably well with measurements made so far, were then used as the basis to determine the location of the dripline, including theoretical uncertainties. The researchers are currently pushing their work to heavier elements. This extended map will ultimately provide valuable input for simulations of the formation of neutron-rich isotopes, such as those created during neutron star mergers.

Marric Stephens is a Corresponding Editor for Physics based in Bristol, UK.

\section{REFERENCES}

1. S. R. Stroberg et al., "Ab initio limits of atomic nuclei," Phys. Rev. Lett. 126, 022501 (2021). 